\title{
Análisis de la evolución de la situación laboral de las mujeres marroquíes ${ }^{1}$
}

\author{
Evolution in employment situation \\ of Moroccan women
}

Khadija Yahya·khadijayahya8@gmail.com

UNIVERSIDAD DE DEUSTO

GRUPO DEUSTO VALORES SOCIALES

Recibido: 23-02-2016

Aceptado: 18-05-2016

\section{Resumen}

El presente artículo analiza la situación laboral de las mujeres marroquíes: desde la primera mitad del siglo XX hasta la década del dos mil. La situación laboral de las mujeres marroquíes ha conocido un notable avance -sobre todo con la incorporación, cada vez más, de las mujeres al mundo laboral- gracias a los cambios que está conociendo la realidad social de estas mujeres. Sin embargo, obstáculos como el género y la disparidad campo/ ciudad siguen presentando grandes desafíos para la sociedad y el Estado marroquí, e impiden su plena inserción laboral.

Palabras clave: Mujeres marroquíes, mundo laboral, género, “petites bonnes”, Marruecos

\section{Abstract}

This article analyzes the employment situation of Moroccan women: from the first half of the twentieth century to the early two thousand. The employment situation of Moroccan women has known remarkable progress -especially with the incorporation, more and more of women to the world of work - thanks to changes that is knowing the social reality of these women. However, obstacles such as gender and the disparity field/City continue presenting major challenges for society and the Moroccan State, and prevent its full employment.

Key words: Moroccan women, world of employment, gender, "small maids", Morocco 


\section{INTRODUCCIÓN}

Hablar de las mujeres de Marruecos, en cualquiera de sus aspectos, no es tarea fácil, debido, sobre todo, a la heterogeneidad de la población femenina de un país en el cual conviven estructuras familiares casi tribales y patriarcales al lado de otras de corte moderno (López, 2003: 101). En Marruecos conviven varias estructuras sociales pertenecientes a distintas épocas históricas. Notamos supervivencias de la Edad Media coexistiendo con otras correspondientes a la actualidad. Según Paul Pascon, se trata de una 'Societé Composite', en el seno de la cual conviven todas las contradicciones armoniosamente debido a su carácter pluralista y su capacidad de evolución (Chafai, 1998: 36).

En esta sociedad caracterizada por la herencia de referencias contradictorias, las mujeres marroquíes se encuentran en el centro de los desafíos de una sociedad donde conviven dos tipos de discursos: el tradicional, basado en la identidad, y el que defiende los valores democráticos modernos (Chafai, 1998: 54). Además, estas mujeres viven y actúan dependiendo de la realidad a la que pertenecen. De hecho, existen distintas realidades relacionadas con varios niveles como la clase social, el nivel de instrucción y de adquisición del saber, de una autonomía económica, el espacio geográfico (ciudad-campo), de su capacidad de tomar una decisión con respecto a elecciones personales o colectivas, y la conciencia de sus derechos (Chafai, 1998: 36).

En la primera mitad del siglo XX, el perfil de la mayoría de las mujeres marroquíes era: amas de casa o esclavas, analfabetas, se les prohibía salir de casa -salvo casos muy raros-, guardadoras fieles de la tradición y de los valores dominantes, supersticiosas, y privatizadas del derecho a elegir sus futuros esposos (Chafai, 1998: 41). En las últimas décadas, han ocurrido muchos cambios en la realidad de las mujeres marroquíes sobre todo en los medios urbanos donde se concentra la mitad de la población (Chafai, 1998: 42). Se admite el hecho de que puedan acceder a todos los rangos jerárquicos y enfrentarse a tareas muy diversas en todos los sectores de actividad, ya sean públicos o privados (Soriano y Santos, 2002: 173). También, se asiste a un descenso de la tasa de fecundidad (Zirari, 2010: 282; Khachani, 2011: 6), un retroceso de la edad media en lo referente a contraer el primer matrimonio (Zirari, 2010, 282; Soriano y Santos, 2002: 179; El Harras, 2004: 42; Soriano, 2004: 138), acceso a la educación, a la formación y al trabajo remunerado (Zirari, 2010: 282), tienen un margen de libertad más amplio a la hora de escoger cónyuge, e intervienen de un modo más real en las decisiones a tomar en la economía doméstica (Soriano y Santos, 2002: 179). Todos estos factores atestiguan las significativas transformaciones sociales y culturales en la vida de las mujeres marroquíes (Zirari, 2010: 282).

Es de subrayar que las mujeres marroquíes de hoy en día están presentes en todos los espacios públicos; esto muestra el cambio radical que ha conocido su situación en las últimas décadas, aunque los valores y símbolos reflejan aún una mentalidad que resiste a ese cambio y que quiere encerrarlas dentro del espacio privado (Chafai, 1998: 35). Es de destacar que los cambios que está conociendo la situación de las mujeres marroquíes son debidos al nuevo Código de la Familia "Mudawana” (2004). Las enmiendas del Código incluyen el 
derecho a decidir sobre cuestiones legales sin la tutela de un hombre, igual responsabilidad en el hogar y para con los hijos, y necesidad del consentimiento tanto del marido como de la mujer para disolver un matrimonio (Green, 2008: 78; CEAR, 2013: 118). Por otro lado, ha favorecido un desbloqueo en la situación política de las mujeres, así como ha cambiado el comportamiento de los partidos políticos; esto último se percibe por una diputada que ha sido designada presidente de un grupo parlamentario (Benlabbah, 2008: 104).

Este trabajo es una exposición teórica de la realidad socio-laboral de las mujeres marroquíes. El objetivo planteado es analizar la evolución de la situación laboral de estas mujeres desde la primera mitad del siglo XX hasta la década del dos mil, a través del análisis de fuentes secundarias, tanto bibliográficas como cuantitativas. La estructura del trabajo es la siguiente: en el segundo apartado se analiza la situación laboral de las mujeres marroquíes desde la primera mitad del siglo XX hasta los años noventa. El tercer apartado consiste en exponer un panorama de la situación laboral femenina marroquí en la década del dos mil. El cuarto apartado trata el fenómeno de las niñas trabajadoras domésticas: "Les petites bonnes”.

\section{LA SITUACIÓN LABORAL DE LAS MUJERES MARROQUÍES DESDE LA PRIMERA MITAD DEL SIGLO XX HASTA LOS AÑOS NOVENTA}

En la primera mitad del siglo XX, la mayoría de las mujeres marroquíes eran amas de casa o esclavas. Debido a la pobreza, un pequeño porcentaje de mujeres se vieron obligadas a trabajar fuera de casa y se dedicaban en la mayoría de los casos al servicio doméstico en las casas de los colonos franceses o españoles, o en algunas fábricas u hospitales. Más tarde, la aceleración de una ligera industrialización al término de la Guerra Mundial, hizo que se incorporasen algunas mujeres -mayoritariamente pobres- al mundo laboral (Masson, 1934 en Chafai, 1998: 36). A pesar de las múltiples actividades que desempeñaron en el mercado laboral, no se valoró su presencia en el proletariado marroquí hasta 1945 (Mernissi, 1987: 73 en Aixelá, 2000: 204).

En relación al estado civil de las mujeres trabajadoras, es de señalar -según una encuesta realizada en Casablanca por Barón (1956, citado en Aixelá, 2000: 205)- que el 16\% de las asalariadas eran solteras, el $43 \%$ casadas y el $41 \%$ viudas o divorciadas. Eran mujeres sin marido o con éste en el paro o ausente las que asumían el trabajo asalariado. Según Belarbi (1993: 19 en Aixelá, 2000: 205), la población activa femenina se caracterizaba por tener empleos estacionales y depender de la demanda, así como por su alta tasa de analfabetismo.

La época de la Independencia de Marruecos marcó la diferencia en lo referente al trabajo femenino. Las mujeres marroquíes han conquistado el mundo laboral sin o con poca formación (Chafai, 1998: 36). Su incorporación al mercado laboral en esta etapa consistió en ocupar puestos de trabajo de especial dureza y a cambio de salarios muy inferiores a los de los hombres, con la excusa de que el dinero obtenido tiene como única finalidad satisfacer sus caprichos (Ajaaouani, 2012: 3). 
Este considerable avance en materia de la ocupación femenina reflejaba los cambios que conocía la sociedad marroquí durante los primeros y posteriores años de la Independencia, como, por ejemplo, la demanda creciente de los parientes a la escolarización de sus hijas y el desarrollo de la educación -entre otros- que han permitido el acceso de las mujeres al modo de producción moderna (Chafai, 1998: 36). En 1952 se registraron 160.000 mujeres activas; de 1960 a 1982 la población activa femenina pasó de 301.000 a 1.181.000, multiplicándose por cuatro en veinte años (Gráfico 1).

Gráfico 1. Población activa femenina marroquí (1952/1960/1982)

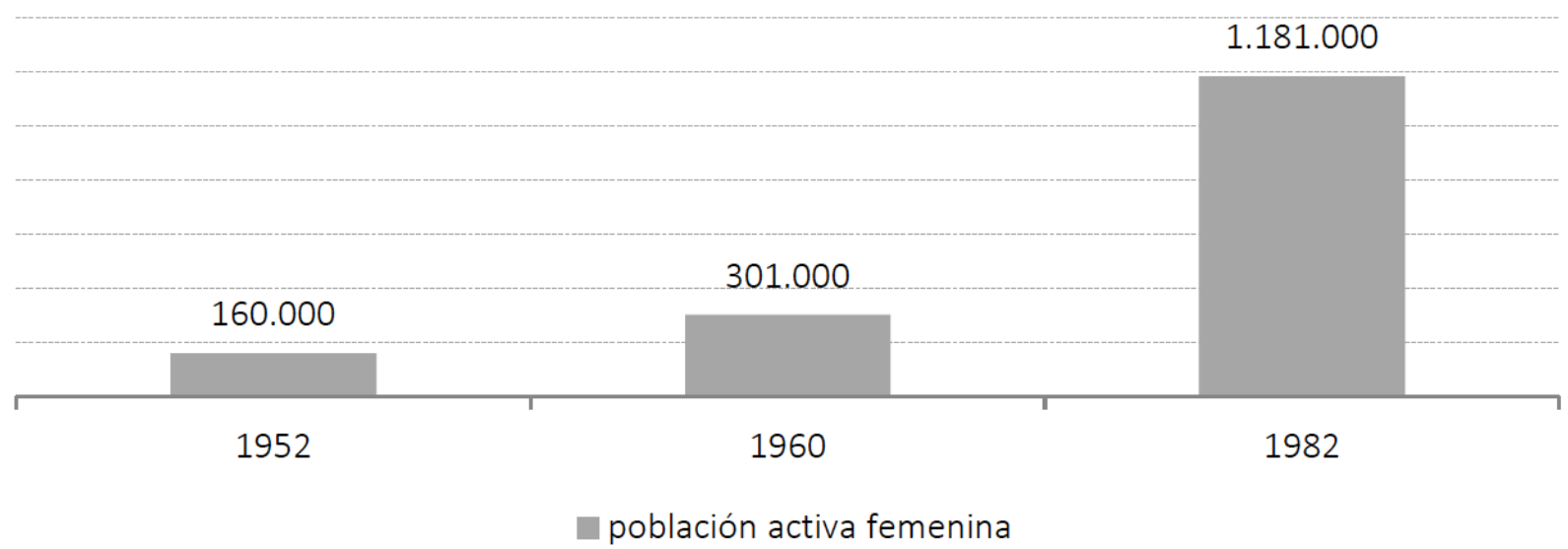

Fuente: Elaboración propia a partir de Chafai (1998).

El total bruto de la actividad femenina pasó de 5,4\% en 1960 a 8,0\% en 1971, y a 11,6\% en 1982 (Aicha Belarbi: 1991 en Chafai: 1998: 36, 37) (Gráfico 2). Entre 1982 y 1999, la tasa de feminización de la población activa ha pasado del 24,3\% al 29,2\% en el medio urbano y del $16,1 \%$ al 38,1\% en el medio rural (Encuestas Nacionales sobre el Nivel de Vida en Hogares (1998-1999) en López: 2003: 103) (Tabla 1).

Gráfico 2. Tasa de actividad femenina marroquí (1960/1971/1982) (\%)

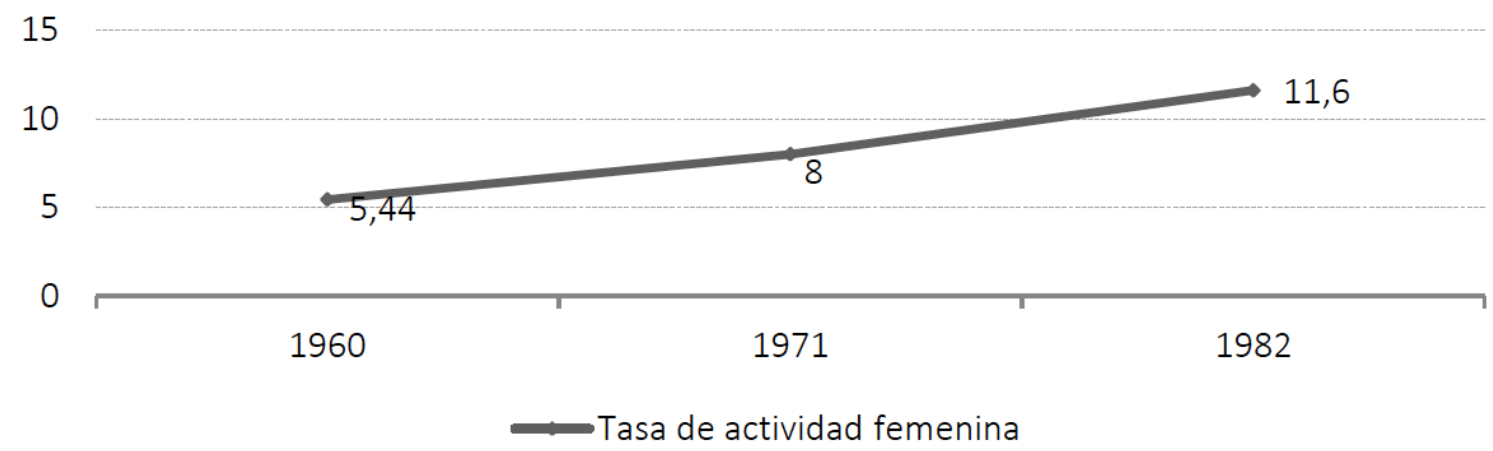

Fuente: Elaboración propia a partir de Chafai (1998).

Tabla 1. Tasa de feminización de la población activa marroquí (1982/1986/1999) (\%)

\begin{tabular}{llll} 
& 1982 & 1986 & 1999 \\
Medio urbano & 24,3 & 26,0 & 29,2 \\
\hline Medio rural & 16,1 & 43,0 & 38,1 \\
\hline
\end{tabular}

Fuente: Elaboración propia a partir de López (2003) y Chafai (1998). 
Según Iván Martín (2008: 64), la tasa de actividad femenina en Marruecos que había experimentado un aumento lento pero sostenido desde los años ochenta llegó a un máximo del $30,4 \%$ de las mujeres en edad de trabajar en 1999, para descender de nuevo hasta el 28,4\% en 2004. Esta evolución, según este mismo autor, tiene su origen, por un lado, en la fluctuación de la actividad agrícola, especialmente importante en Marruecos; y por otro lado, en el descenso del trabajo femenino en la industria, especialmente, en la industria textil como consecuencia del desmantelamiento del Acuerdo Multifibras de la Organización Mundial del Comercio, y la consiguiente retirada de muchas mujeres del mercado laboral. Esto último pone de evidencia que las mujeres, en general, y el empleo femenino, en particular, son las más afectadas de manera directa por los impactos negativos de la globalización y la liberalización económica.

Por otra parte, según el informe Femmes et condition feminine de la Direction des Statistiques (1994 en Chafai, 1998: 37), en lo que respecta a los grupos de edad de las mujeres trabajadoras, el análisis de la actividad de la población femenina de edad comprendida entre 25 y 44 años refleja un aumento regular de la inserción de las mujeres en el mundo laboral entre 1985 y 1990, y un descenso de 1991 a 1993. De hecho, de 1985 a 1990, estas tasas pasaron de $32,4 \%$ a 37,3\% para las de 25 a 29 años; 29,5\% a 34,5\% para las de 30 a 34 años; 24,0\% a 27,6\% para las de 35 a 39 años; y de 20,7\% a 24,8\% para las de 40 a 44 años, mientras que de 1991 a 1993 el descenso de la tasa de actividad ha afectado al conjunto de los grupos de edad mencionados. En efecto, estas tasas han descendido de 38,4\% a 34,9\% (25-29 años); de $35,6 \%$ a $31,3 \%$ (30-34); de $27,8 \%$ a $26,3 \%$ (35-39 años); y de $24,2 \%$ a $23,2 \%$ (40-44 años); es decir, respectivamente una disminución de 9,1\%; 12,1\%; 5,4\% y 4,1\% (Tabla 2).

Tabla 2. Tasa de la actividad de la población femenina marroquí por grupos de edad (\%)

\begin{tabular}{l|llll} 
& 1985 & 1990 & 1991 & 1993 \\
\hline $25-29$ & 32,4 & 37,3 & 38,4 & 34,9 \\
$30-34$ & 29,5 & 34,5 & 35,6 & 31,3 \\
\hline $35-39$ & 24,0 & 27,6 & 27,8 & 26,3 \\
\hline $40-44$ & 20,7 & 24,8 & 24,12 & 23,2 \\
\hline
\end{tabular}

Fuente: Elaboración propia a partir de Chafai (1998).

El motivo de tal disminución, sobre todo en los puestos de trabajo en el sector público se debe a que estos años coincidieron con los últimos años de la aplicación del Programa de Ajuste Estructural (el Estado redujo los puestos consagrados a la función pública: 50.000 puestos en 1983 descendieron a 12.000 según la ley financiera de 1995). De este modo, las mujeres se convirtieron en "chivo expiatorio" de la crisis económica. Dadas estas circunstancias, las mujeres del medio urbano empezaron a buscar otras alternativas en el sector privado. Como consecuencia, a finales de los años noventa, Marruecos contaba con más de 10.000 mujeres patronas (dueñas de sus empresas) que ofrecían trabajo (Chafai, 1998: 37).

En lo referente a los sectores que usaban por esas fechas la mano de obra femenina, cabe señalar, especialmente, el de la agricultura y la industria agroalimentaria, el sector textil, y el de servicios (doméstico, turístico y administrativo). En cuanto al sector público, las mujeres representaban 1/5 de los funcionarios del Estado (20,3\%); la mayoría de ellas estaban 
insertadas en las escalas más bajas de la función pública: sólo 4,9\% ocupaban altos cargos públicos (Chafai, 1998: 37).

Se puede distinguir entre dos tipos de contextos sociales en los que las mujeres desarrollan su función como trabajadoras: el urbano y el rural. En el contexto rural, la mujer realiza muchas actividades, ya sea dentro o fuera del hogar familiar. Estas actividades son poco reconocidas y no remuneradas; se realizan normalmente dentro de un contexto de explotación familiar.

En el contexto urbano, las mujeres desarrollan, habitualmente, empleos que se realizan fuera de la economía familiar y del espacio doméstico, y se combinan con sus obligaciones familiares y del hogar. La incorporación de estas mujeres al mercado laboral se produce tanto en la esfera formal como informal. En la formal destacan las funciones de administrativa, enfermera, profesora, cosedora y despiezadora en una fábrica, entre otros empleos. En la esfera informal destacan los trabajos de servicio doméstico o de confección en su hogar o en una fábrica (Aixelá, 2000: 212).

Son diversas las razones por las que las mujeres se incorporan a la economía informal. Por una parte, según Afifi y Bendagha (1986: 29 citadas en Aixelá, 2000: 219), este sector representa una salida profesional para las mujeres analfabetas. Por otra parte, las actividades informales que realizan algunas mujeres en su hogar interesan a aquellos esposos que no desean asumir un reparto de las responsabilidades domésticas con sus esposas. Además, las actividades informales constituyen una solución tanto para algunas mujeres como para los hombres de su grupo en tanto que evitan confundir su movilidad por los espacios público y privado y buscan la invisibilidad de su trabajo (Aixelá: 2000: 219, 220). Responde entonces a las necesidades de aquellas familias que desean un sobresueldo pero sin cuestionar ni el género propuesto desde el parentesco ni el reparto de actividades que se desprende de la estructura familiar (Mahfoudh, 1990: 169 en Aixelá, 2000: 220).

Es más, dependiendo del sector de trabajo -formal o informal-, se ve cuestionada la construcción del género. De hecho, los trabajos en los sectores formales, donde se insertan trabajadoras cualificadas y cuyos sueldos son altos, son los que provocan que se dude explícita y públicamente de la actividad masculina como proveedor de la familia. De lo contrario, los trabajos que se desarrollan en el sector informal cuestionan poco la construcción del género porque se interpretan como una aportación económica poco elevada, transitoria, y que se realiza en casa combinándose con las tareas domésticas, sin distorsionar así los repartos espaciales según el sexo (Aixelá, 2000: 229).

\section{EL PANORAMA LABORAL FEMENINO EN LA DÉCADA DEL DOS MIL}

En la década del dos mil, la participación femenina en la actividad económica marroquí se inscribe en una tendencia ascendente (Khachani, 2011: 6). Sin embargo, la feminización de la función pública y el aumento del número de mujeres no implican la existencia de equi- 
dad e igualdad de oportunidades respecto al acceso a las responsabilidades y las funciones. Todavía existen obstáculos que impiden a las mujeres llegar a las esferas de toma de decisiones en igualdad con los hombres, a pesar de la existencia de las leyes que lo estipulan (Zirari, 2010: 284).

Hoy en día, las mujeres presentan únicamente el 25\% de la población activa marroquí y su tasa de empleo es inferior a la de los hombres (Zirari, 2010: 287). Es de destacar, según datos del Informe sobre Desarrollo Humano 1999, realizado por el PNUD (en Díez, 1999: 66), que en los países en desarrollo, las mujeres constituían menos de un séptimo del número total de altos funcionarios y el porcentaje era inferior al 5\% o inexistente en 55 países. Es más, a comparación con los países de Oriente Medio y Norte de África (MENA), la participación de las mujeres marroquíes en el mundo laboral sigue presentando deficiencias y se considera una de las más bajas de entre ellos (Zirari, 2010: 287).

Es de señalar, también, que en los países del Magreb -sobre todo en Marruecos, Argelia y Túnez- la tasa de actividad para las mujeres es entre dos y tres veces inferior a la de los hombres, según el Banco Mundial. Aunque han aumentado significativamente en las últimas décadas, siguen por debajo de las tasas de actividad femenina de cualquier otra región del mundo, y mantienen casi 50 puntos porcentuales de diferencia con la tasa de actividad de los hombres (Martín, 2008: 64). Aun así, el acceso de las mujeres marroquíes al trabajo remunerado con la asunción de responsabilidades políticas y públicas constituye un indicador importante del cambio en Marruecos: 'Nos encontramos ante un Marruecos, sin duda, que se mueve y donde la tendencia se dirige hacia la construcción de una nueva identidad y una nueva relación entre hombres y mujeres' (Zirari, citada en DUPO: 22.07.10).

Según un informe publicado por el Haut-Commissariat au Plan (2013: 15, 16), alrededor de 1,2 millón de las familias marroquíes de un total de 6,8 millones (casi 17,7\%) estaban encabezadas por mujeres en 2012. Según el lugar de residencia, la proporción de hogares encabezados por éstas representan un alto porcentaje en zonas urbanas (19,6\%) frente a $14,1 \%$ en zonas rurales. Más de la mitad de las mujeres cabezas de familia $(55,1 \%)$ son viudas, $26,6 \%$ están casadas y 11,1\% están divorciadas frente a sólo 6,8\% de solteras. Además, las mujeres cuya edad es de menos de 35 años representan el 13\% frente a 37,2\% de las mujeres que tienen más de 60 años. El 86\% de estas mujeres no tiene título académico, y sólo $4,4 \%$ de ellas tiene titulación académica superior. En las zonas rurales estos porcentajes representan $97,1 \%$ y $0,4 \%$ respectivamente. La misma fuente añade que la cuarta parte de estas mujeres trabaja y más de 7 de cada 10 no son activas. En las zonas rurales, que están dominadas por el sector agrícola, la proporción de mujeres trabajadoras que sostienen a sus familias asciende a $36,2 \%$ frente al $63,1 \%$ de las mujeres que no son activas (Tabla 3). 
Tabla 3. Mujeres marroquíes cabezas de familia, según lugar de residencia, estado civil, edad y nivel de formación (2012) (\%)

\begin{tabular}{|c|c|c|c|c|c|c|c|c|c|c|c|}
\hline \multicolumn{2}{|c|}{ Lugar de residencia } & \multicolumn{4}{|c|}{ Estado civil } & \multicolumn{2}{|c|}{ Edad } & \multicolumn{4}{|c|}{ Nivel de formación } \\
\hline \multirow{2}{*}{$\begin{array}{l}\text { Zonas } \\
\text { urbanas }\end{array}$} & \multirow{2}{*}{$\begin{array}{l}\text { Zonas } \\
\text { rurales }\end{array}$} & \multirow{2}{*}{ Casada } & \multirow{2}{*}{ Viuda } & \multirow{2}{*}{ Divorciada } & \multirow{2}{*}{ Soltera } & \multirow{2}{*}{$\begin{array}{l}\text { Menos } \\
\text { de } \\
35 \text { años }\end{array}$} & \multirow{2}{*}{$\begin{array}{l}\text { Más } \\
\text { de } 60 \\
\text { años }\end{array}$} & \multicolumn{2}{|c|}{$\begin{array}{l}\text { Sin titulación } \\
\text { académica }\end{array}$} & \multicolumn{2}{|c|}{$\begin{array}{c}\text { Con titulación } \\
\text { académica }\end{array}$} \\
\hline & & & & & & & & $\begin{array}{l}\text { Zonas } \\
\text { urbanas }\end{array}$ & $\begin{array}{l}\text { Zonas } \\
\text { rurales }\end{array}$ & $\begin{array}{l}\text { Zonas } \\
\text { urbanas }\end{array}$ & $\begin{array}{l}\text { Zonas } \\
\text { rurales }\end{array}$ \\
\hline 19,6 & 14,1 & 26,6 & 55,1 & 11,1 & 6,8 & 13,0 & 37,2 & 86,0 & 97,1 & 4,4 & 0,4 \\
\hline
\end{tabular}

Fuente: Elaboración propia a partir del Haut Commissariat au Plan (2013).

Por otra parte, es de destacar que la mayoría de las mujeres marroquíes activas son analfabetas (58,9\% frente a 32,9\% de hombres) (Zirari, 2010: 287), carecen de la preparación para el mercado laboral nacional, y de una adecuada educación y formación profesional (Soriano y Santos, 2002: 174). Respecto al mundo rural, el índice de analfabetismo entre las mujeres alcanza el 84\% (Zirari, 2010: 287), y la mayoría de las que están activas no reciben remuneración alguna por considerar su trabajo como ayuda doméstica o aprendices (Zirari, 2010: 287; Ramírez, 2004: 39).

Es de señalar, también, según el informe del Haut-Commissariat au Plan (2013: 6, 7), que las mujeres marroquíes trabajadoras son relativamente jóvenes $(42,4 \%$ tienen menos de 35 años). En el medio rural, la implicación de las chicas jóvenes de menos de 25 años en las actividades agrícolas hace que su tasa en el empleo femenino se aproxime al 20\%. Respecto al medio urbano, donde los esfuerzos para la escolarización de las chicas jóvenes son relativamente más importantes, esta proporción es de sólo un 10\%. Es de subrayar que en 2012, un 73,5\% de mujeres rurales ha declarado haber accedido al mercado laboral a una edad inferior a 15 años (frente a sólo un 11,8\% entre las mujeres urbanas). Sin duda, esta realidad tiene unos efectos negativos sobre la escolarización de niñas y niños en el medio rural, sobre todo con respecto a las niñas.

Los sectores donde están ocupadas las mujeres marroquíes son esencialmente la "agricultura, bosque y pesca” (59,9\%), “servicios” (27,0\%) y “la industria” (11,8\%). Esta estructura de trabajo según los sectores de actividad esconde las disparidades por lugar de residencia (rural/urbano). Además, las mujeres activas ocupadas trabajan esencialmente en el sector privado (90,9\%), en que más de la mitad está ocupada en las explotaciones agrícolas (59,5\%). En el medio rural, más de tres cuartos de mujeres activas ocupadas son obreras u obreras en la agricultura o la pesca (75,2\%), y 17,9\% son explotadoras agrícolas, pescadoras, guardias forestales o cazadoras. Por otra parte, en el medio urbano, alrededor de $45 \%$ de mujeres ejercen como artesanas u obreras en pequeños trabajos, y casi un $40 \%$ lo hace como ejecutivo medio o empleadas (Haut-Commissariat au Plan, 2013: 9, 10) (Gráfico 3). 
Gráfico 3. Los sectores de actividad donde están ocupadas las mujeres marroquíes (2012) (\%)

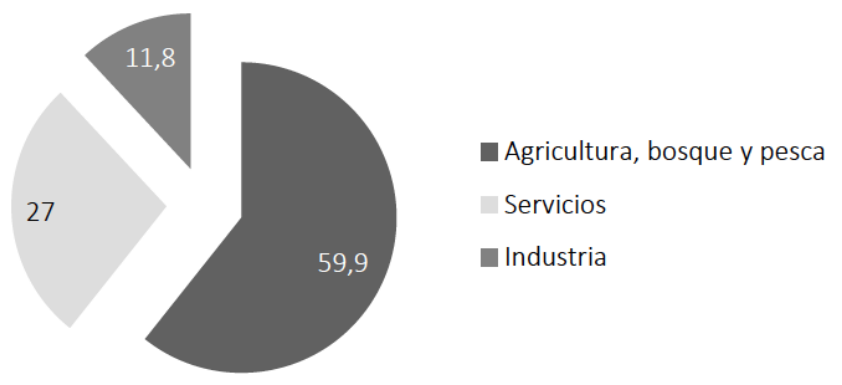

Fuente: Elaboración propia a partir del Haut Commissariat au Plan (2013).

Respecto al desempleo, las mujeres activas jóvenes -de edad comprendida entre 25 y 34 años- se ven más sometidas al paro (Zirari, 2010: 287). De hecho, según Khachani (2011: 8), en el año 2008, las tasas más altas de desempleo se registraron en los grupos de edad comprendida entre 15-24 (16,1\%) y 25-34 años (15,5\%). El aumento de desempleo de estas mujeres refleja la gran fragilidad en cuanto a su inserción laboral (Zirari, 2010: 287). En 2009, la tasa de desempleo femenino ha sido de $9,8 \%$ y excede los $20 \%$ registrados en el medio urbano: una mujer activa sobre cinco. Estas tasas fueron mucho más altas en 1999: 13,3\% y 27,6\% respectivamente (Khachani, 2011: 7). Según la el Haut Commisariat au Plan (2015), en el segundo trimestre del año 2015 la tasa de feminización de la población activa en situación desempleo es de 28,1\% (26,9\% en el mismo periodo del 2014); y la tasa de desempleo de mujeres es de 9,0\% (9,0\% en el mismo periodo de 2014) (Gráfico 4).

Gráfico 4. Tasa de desempleo de las mujeres marroquíes (1999/2009/2014/2015) (\%)

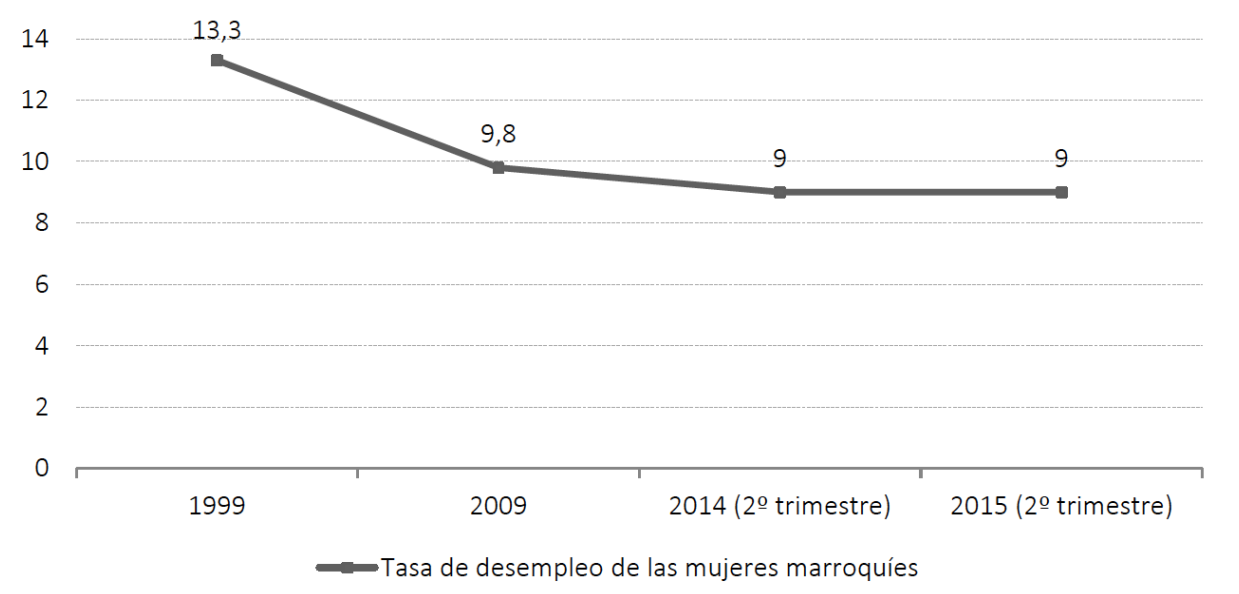

Fuente: Elaboración propia a partir de Khachani (2011) y el Haut Commissariat au Plan (2015).

Es de destacar que las mujeres universitarias son las más afectadas por el desempleo: 41\%; es decir, casi el doble de la tasa global (22,9\%), incluidos los dos sexos (Khachani, 2011: 8). En 2012, según el Haut-Commissariat au Plan (2013: 12), las mujeres en situación de desempleo se caracterizan esencialmente por su edad joven y su cualificación. De hecho, casi ocho mujeres de diez son tituladas. Además, tres cuartos de ellas $(75,1 \%)$ se encuentran desempleadas desde más de un año, y 64\% son demandantes de empleo por primera vez.

Según Iván Martín (2008: 65, 66), la progresiva feminización de la escolarización universitaria y de la población activa en los países de Magreb se está traduciendo en la feminización 
del desempleo y no del empleo. Tanto en Túnez como en Marruecos y en Argelia, cuanto mayor es el nivel de instrucción de la mujer mayor es la tasa de desempleo, algo que también sucede en el caso de los hombres pero en menor medida. Para el autor, no se trata de un “desempleo de inserción” puesto que el desempleo de larga duración no cesa de aumentar en estos países: en Marruecos, casi tres cuartas partes de las mujeres desempleadas llevan más de un año buscando trabajo frente a dos de cada tres hombres, y muchas mujeres acaban retirándose del mercado laboral sin haber llegado nunca a tener un empleo formal. Como única salida a esta situación para las mujeres que pretenden obtener una emancipación económica es la administración pública -que cada vez crea menos empleos- o la emigración.

Según el informe Le travail des maghrébins. L’autre enjeu (2006: 50-57, en Martín, 2008: 66), las políticas activas de empleo que aplican los Estados del Magreb para facilitar la inserción laboral de los jóvenes no cuentan con programas específicos dirigidos a las mujeres cualificadas a pesar de la importancia que tiene el gasto en estas políticas (nada menos que un 1,5\% del PIB en Túnez, 0,5\% del PIB en Marruecos y 0,6\% en Argelia). Esto agrava la situación de las mujeres cualificadas en un mercado de trabajo discriminatorio. Dichas políticas parecen venir a reforzar esta auténtica división sexual del trabajo en estos países que, a su vez, es uno de los principales mecanismos de subordinación de las mujeres.

Por otro lado, es de destacar un dato muy importante referente a la prioridad que dan las mujeres mediterráneas -Marruecos incluido- al trabajo cuando éste es escaso; es decir, saber qué percepción tienen sobre la prioridad que deberían tener los hombres de acceso a los puestos de trabajo, cuando éstos son escasos. Según un estudio llevado a cabo por World Values Survey (Cebolla, 2008: 83, 84), en la mayoría de países del sur del Mediterráneo (Egipto, Jordania y Marruecos), incluso las mujeres de menos de 24 años darían prioridad a los hombres en el acceso al mercado de trabajo. Las únicas excepciones se encuentran entre las argelinas y las turcas.

En este sentido, la valorización del trabajo por parte de las mujeres marroquíes se da en el colectivo de mujeres profesionales, pero no es tan evidente entre el colectivo de mujeres que ocupan trabajos no cualificados y muy mal remunerados -que les hacen sentir que los trabajos ejercidos están siendo infravalorados y son innecesarios- que a la menor ocasión los dejan: en el caso de las solteras puede ser un matrimonio que las sitúe en una mejor posición en la escala socioeconómica, como si la participación de las mujeres solteras en la fuerza de trabajo fuera algo que es deseable que sea transitorio (Ramírez, 1998: 92).

En lo que respecta al tema de la no remuneración del trabajo de las mujeres, es de señalar que esto último constituye un fenómeno de carácter general que alcanza hasta a las mujeres en el medio urbano. Las mujeres marroquíes en activo sin remuneración representan cerca del 31\% del conjunto del colectivo femenino ocupado (84\% para las mujeres rurales). Los efectos negativos de este fenómeno se reflejan en la no posibilidad de afianzar la autonomía femenina y en su incapacidad de negociación en la familia. Otro aspecto que ataña el salario de las mujeres activas es la no igualdad de salarios entre éstas y los hombres. El predominio 
de la desigualdad salarial en relación al género afecta hasta a las ocupadas en la función pública -que representan un tercio del personal del Estado-, que sólo perciben el 29\% de la masa salarial anual bruta (Zirari, 2010: 287).

Respecto a su estado civil, es de señalar que este último ocupa un papel prioritario en relación a la realización de un trabajo remunerado: casi la mitad de las mujeres ocupadas son solteras. Este hecho significa que el matrimonio conforma un obstáculo más para el acceso de las mujeres al mundo laboral, esencialmente por herencia cultural que consiste en que el trabajo de la mujer casada fuera de hogar deshonra a la familia por significar que su marido no ha podido mantenerla (Soriano y Santos, 2002: 174).

En cuanto a la protección social, las mujeres marroquíes trabajadoras presentan una tasa relativamente baja de sindicalización, sufren acoso sexual en el ámbito laboral -que en la mayoría de los casos no se denuncia por miedo a perder el puesto de trabajo-, gran mayoría de ellas no se halla cubierta por el régimen del Seguro Médico Obligatorio (AMO) creado en el 2006 (Zirari, 2010: 287). Además, muchas mujeres no disfrutan de la asistencia sanitaria a pesar de tener un puesto de trabajo (Ajaaouani, 2012: 10); dentro de las cuales, están las mujeres trabajadoras en el hogar, en el servicio doméstico, y en el sector tradicional, que no gozan de ninguna protección social ni médica. Los hombres se benefician más que las mujeres del sistema de protección social. De hecho, en cuanto a la cobertura de jubilación, estas desigualdades se hacen patentes debido a que las mujeres trabajan a tiempo parcial y ganan menos que los hombres o desempeñan trabajos precarios con contratos temporales, lo que repercute negativamente en las cuotas y las prestaciones de jubilación que suelen ser menores que las de los hombres (Zirari, 2010: 287).

En este sentido, y según el Haut-Commissariat au Plan (2013: 11), el trabajo en Marruecos de manera global se caracteriza aún por una cobertura limitada respecto a la protección social. De hecho, la mayoría de las/os trabajadores (80,9\%) no están afiliadas/os a ningún sistema de cobertura sanitaria ( $81,6 \%$ en mujeres frente a $80,7 \%$ en hombres). En el medio urbano, un 53,3\% de mujeres en activo no tiene ninguna cobertura sanitaria frente a $69,6 \%$ en hombres. En el medio rural, la casi totalidad de mujeres ejerce las actividades sin ninguna cobertura sanitaria (98,8\% frente a 94,2\% en hombres). Asimismo, el hecho de tener un título aumenta la oportunidad de acceder a un empleo, asegurando la cobertura sanitaria. Así, la tasa de activos ocupados afiliados a un sistema de cobertura sanitaria pasa de 4,1\% para los que no disponen de títulos a $81,2 \%$ para los titulados de nivel superior (Tabla 4).

Tabla 4. Afiliación al sistema de cobertura sanitaria, según sexo y titulación académica (2012) (\%)

\begin{tabular}{|c|c|c|c|c|c|c|}
\hline & \multicolumn{2}{|c|}{ Mujeres } & \multicolumn{2}{|c|}{ Hombres } & \multirow{3}{*}{$\begin{array}{c}\text { No } \\
\text { tituladas/os }\end{array}$} & \multirow{3}{*}{$\begin{array}{c}\text { Tituladas/os } \\
\text { superiores }\end{array}$} \\
\hline & $\begin{array}{l}\text { Medio } \\
\text { urbano }\end{array}$ & $\begin{array}{l}\text { Medio } \\
\text { rural }\end{array}$ & $\begin{array}{l}\text { Medio } \\
\text { urbano }\end{array}$ & $\begin{array}{l}\text { Medio } \\
\text { rural }\end{array}$ & & \\
\hline $\begin{array}{l}\text { Trabajadoras/es no afiliadas/os a } \\
\text { ninguna cobertura sanitaria }\end{array}$ & 53,3 & 98,8 & 69,6 & 94,2 & & \\
\hline $\begin{array}{l}\text { Trabajadoras/es afiliadas/os a algún } \\
\text { sistema de cobertura sanitaria }\end{array}$ & & & & & 4,1 & 81,2 \\
\hline
\end{tabular}

Fuente: Elaboración propia a partir del Haut Commissariat au Plan (2013). 
El derecho laboral marroquí equipara al hombre y a la mujer respecto al trabajo y a la seguridad social mediante los tres reglamentos que regulan la vida laboral: Reglamentación General del Trabajo, Régimen de Salario Agrícola y Estatuto de la Función Pública. Sin embargo, ese igualitarismo queda parcialmente anulado por otra serie de disposiciones con las que choca de forma evidente como la necesidad de autorización del marido para firmar un contrato de trabajo o para realizar alguna actividad comercial para una mujer casada (López, 2003: 103).

Según el informe EMIAT (1993), el desprestigio y los bajos sueldos del trabajo remunerado para mujeres se han resuelto con la emigración al extranjero, y en los últimos años con las migraciones interiores. Según el informe, se constataba que entre los emigrantes interiores había más mujeres que hombres: 51,6\% frente a 48,3\%. El porcentaje de las mujeres no casadas aumentaba entre las emigrantes más recientes: de un 33,8\% en el grupo de inmigrantes que llegaron hace más de diez años hasta un 38,2\% en las recientes. Este aumento se daba sobre todo en el porcentaje de solteras que pasaban de $17,7 \%$ entre las emigrantes de larga duración a un 25,5\% entre las que habían llegado en los últimos diez años. Bajaban, sin embargo, los porcentajes de viudas y divorciadas para las recién llegadas. La emigración interior de las mujeres se va desvinculando en los últimos años de la emigración familiar y conyugal por un lado, y de la huida de situaciones de desprestigio social y económico, por otro (Ramírez, 2004: 39).

Además, en estos últimos años, la emigración interior de las mujeres ha creado unas formas inéditas de residencia. Esto es, las mujeres que llegan a los grandes núcleos procedentes de ciudades pequeñas y medianas para trabajar si no tienen parientes que residen ya en ella, comparten pisos. Estas formas de residencia, escasas hasta el momento, en las ciudades marroquíes han hecho que cambie esa imagen de las mujeres que vivían siempre protegidas, vigiladas o legitimadas por alguna mujer de edad o algún pariente varón (Ramírez, 2004: 39).

Respecto a la otra opción, la inmigración al extranjero, es de señalar cómo se convierte en una forma de movilidad social para las mujeres urbanas y contribuye a la creación de nuevas formas de estratificación social para estas mujeres de manera independiente del estatuto de los hombres (Ramírez, 2004: 40). La percepción por parte de algunas mujeres trabajadoras de los procesos de exclusión que se viven desde el trabajo les lleva a plantearse la opción de construir su identidad fuera del trabajo e incluso a imaginar y planear la emigración hacia otro país del cual tienen referencias personales de progreso (Solís, 2010: 76). Es más, esta opción deduce -en el caso de las mujeres cualificadas, particularmente-, que la obtención de títulos y el reconocimiento de la igualdad de derechos civiles y políticos no basta para garantizar su integración en la vida económica (Martín, 2008: 66).

Es de señalar, en este sentido, según Khachani (2011: 9), que la cultura de la emigración está arraigada entre las mujeres marroquíes incluso desde una edad temprana. De hecho, en una encuesta sobre el trabajo infantil codirigida por el mismo autor, el 13,2\% de las chicas jóvenes han declarado tener como proyecto de futuro emigrar fuera de Marruecos. 


\section{LAS NIÑAS TRABAJADORAS DOMÉSTICAS O “LES PETITES BONNES"}

Marruecos es conocido por tener vivo todavía un sector que parece reflejar dos de las cuestiones que más afectan a las mujeres: su vulnerabilidad como mano de obra -sobre todo si son pobres y menores- y las relaciones de poder entre grupos diferentes; por ejemplo, "les petites bonnes” o el servicio doméstico infantil (Ramírez, 2004: 40).

El trabajo infantil fue un tema tabú durante mucho tiempo en Marruecos. Sin embargo, desde hace una quincena de años, ha tornado como una temática tratada por las autoridades públicas y las asociaciones en el marco de la defensa de los derechos del niño (CEAR, 2013: 131).

El trabajo infantil está prohibido por la ley marroquí. Sin embargo, existen niñas/os trabajadoras/es en diferentes sectores incluso en aquellos que suponen peligro para la salud del menor (Ajaaouani, 2013: 13). Este fenómeno ha conocido un descenso importante durante los años dos mil. De hecho, entre 1999 y 2011, el Haut Commisariat au Plan considera que la proporción de niñas/os trabajadoras/es de entre 7 y 15 años descendió desde un 9,7\% (517.000 niñas/os) hasta 2,5\% (123.000 niñas/os); de entre las/os niñas/os trabajadoras/es, 6 de cada 10 son chicos y 9 de cada 10 trabajan en el medio rural; Además, el 16,6\% sigue acudiendo a la escuela mientras trabaja, el 56,1\% dejó la escuela mientras que el 27\% nunca la frecuentó (CEAR, 2013: 131).

La pobreza constituye uno de los factores determinantes del trabajo de los menores, pero no es el único; también están la separación de los padres, la muerte de uno de ellos o los dos, niños ilegítimos, el abandono de uno de los padres del hogar familiar, el analfabetismo, la ignorancia de los padres y el elevado número de niñas/os en la unidad familiar, entre otros (Ajaaouani, 3012, 10). Según un estudio en cooperación entre el Ministerio de Desarrollo Social, el Ministerio de Trabajo, La Formación Profesional y la Fundación Mundial, el 82\% de los niñas/os trabajadoras/es son niños de familias pobres (Ajaaouani, 2013: 10).

Algunos sectores favorecen el trabajo infantil. En el medio rural, están la agricultura, las actividades forestales o la pesca, mientras que en el medio urbano, el 53,7\% de los niñas/ os trabajadoras/es se encuentra en los servicios; el 42\% de los trabajadores menores de 15 años es empleado de la industria manufacturera y la artesanía como aprendices (formados en mayoría por chicos), mientras que el primer sector de empleo para las niñas en el medio urbano es la industria de la alfombra y otra gran parte de ellas trabaja como "petites bonnes” (trabajadoras domésticas pequeñas) (CEAR, 2013: 132).

Según Lahlou (2001: 1013), en 1999, las niñas marroquíes cuya edad es menor de quince años representaban el 8,7\% de la población ocupada (4,1\% en el caso de los niños). No existen estadísticas precisas sobre el número de "petites bonnes” en Marruecos; aun así, podemos citar la Encuesta Nacional a Indicadores Múltiples y Salud de los Jóvenes (ENIMSJ), llevada a cabo por el Ministerio de la Salud en 2008, que registra a un 36\% del total de niñas/ os trabajadoras/es en el sector doméstico, teniendo en cuenta que las cifras reales pueden ser superiores (CEAR, 2013: 133). 
Además, según un estudio llevado en 2010 por el Colectivo Marroquí para la Erradicación del Trabajo de las "Petites Bonnes”, el 62\% de las niñas trabajadoras en el servicio doméstico tenía entre 13 y 15 años, y el 38\% entre 8 y 12 años. En general, tienen un nivel de escolaridad bajo: 30\% de ellas nunca acudió a la escuela, 49\% la abandonó, 21\% estaba escolarizado pero trabajaba durante las vacaciones. Además, el 43\% dejó la escuela por motivos económicos mientras que el 25\% abandonó los estudios por la distancia entre la escuela y el lugar de residencia. En cuanto a los parientes de las niñas, sólo el 9\% tenía ingresos regulares; además, el 94\% de las madres y el 72\% de los padres eran analfabetos (CEAR, 2013: 134).

Sin embargo, todos los actores preocupados por esta problemática coinciden en apuntar que esta realidad está en declive desde hace unos años como consecuencia de los esfuerzos llevados por ONGs, agencias de las Naciones Unidas, y el gobierno para escolarizar a los niños y concienciar a las familias acerca de los peligros implicados por el trabajo doméstico infantil. Además, el arresto muy mediatizado de una mujer acusada de haber golpeado y matado a una trabajadora doméstica menor de edad en julio de 2011 contribuyó a este proceso de concienciación (CEAR, 2013: 133).

La gran mayoría de estas niñas provienen de las áreas rurales pobres y se dirigen a las grandes ciudades como Casablanca, Rabat, Marrakech, Fes, Agadir, Tánger, etc. (CEAR, 2013: 134; Ajaaouani, 2013: 4, 5). Asumen la responsabilidad de mantener a sus familias en una edad muy temprana -desde los seis años- (Ajaaouani, 2013: 5). Según Naamane y Chakib Guessous (citados en Ajaaouani, 2013: 5, 6), esta situación hace que la mayoría de ellas se escapen del domicilio en donde trabajan para encontrarse en la calle, ya que no pueden regresar al hogar familiar por temor a ser castigadas por los padres por abandonar el trabajo.

El reclutamiento de las niñas implica un intermediario (CEAR, 2013, 134; Ajaaouani, 2013: 6) quien recibe honorarios por parte de los empleadores, pero a veces son los padres quienes tienen que pagar al intermediario si éstos no pueden hacerlo. En este caso, la hija trabaja sin cobrar durante dos o tres meses para pagarlo. En la mayoría de los casos, los intermediarios prometen buenas condiciones laborales -que acaban discrepando con la realidad-, o engañan a la familia sobre la índole del trabajo (CEAR, 2013: 134). Además, les interesa que la niña cambie regularmente de trabajo para percibir más honorarios (CEAR, 2013: 134; Ajaaouani, 2013: 12). Debido a la falta de control por parte del Estado, los intermediarios son los principales beneficiarios del trabajo infantil (Ajaaouani, 2013: 12).

Las tareas que desempeñan las niñas suelen consistir en fregar los platos, lavar la ropa, limpiar el suelo y las alfombras, ir de compras, cuidar a los niños, llevar los hijos a la escuela y recogerlos, servir la comida a los invitados, etc., aunque la asunción de las responsabilidades dependen también de su edad (CEAR, 2013: 134).

"Les petites bonnes" suelen trabajar en condiciones de abuso y explotación debido a la falta de ley que regule este sector. Su sueldo corresponde apenas a un cuarto del salario mínimo del sector industrial (unos 545 dirhams al mes que se entregan directamente a los padres de las niñas); no tienen día libre incluso cuando están enfermas; se levantan las primeras por la mañana y se acuestan las últimas por la noche; muchas veces renuncian a ver a un 
médico cuando están enfermas por miedo a deducirle el empleador los gastos de su sueldo; pueden sufrir hambre; sufren de malas condiciones de acogida (se dan testimonios de niñas obligadas a dormir en una alacena o en el suelo de la cocina en condiciones de frío, etc.) (CEAR, 2013: 135); aislamiento; incomprensión -sobre todo las que hablan tamazight y no entienden árabe- (CEAR, 2013: 135; Ajaaouani, 2013: 6); sufren abusos físicos y verbales cometidos por sus empleadores, así como violencia sexual por parte de los miembros masculinos del hogar (CEAR, 2013: 135).

Ante la explotación a la que se ven sometidas, estas niñas escapan del domicilio donde trabajan y se encuentran solas en la calle donde pueden sufrir todo tipo de violencia: violación, vagabundeo, delincuencia o prostitución (Ajaaouani, 2013: 8). Según Aicha Belarbi (Ajaaouani, 2013: 8), en algunos casos, pueden ser acusadas falsamente de robo por su empleadora como método para castigarla al abandonar el trabajo; esta situación hace que "les petites bonnes” sean futuras madres solteras. Según Naamane y Guessous (citados en Ajaaouani, 2013: 15), hoy en día, en Marruecos el número de menores madres solteras es inquietante. En el año ochenta, 17,1\% de madres solteras tenían menos de 18 años; en los noventa, 30\% de madres solteras tenían menos de 20 años; de 2003 a 2004, de 590 madres solteras recibidas por la asociación INSAF, 24,2\% de ellas tenían 20 años o menos.

\section{CONCLUSIONES}

En los principios del siglo XX, la mayoría de las mujeres marroquíes eran amas de casa o esclavas; trabajaban por motivo de la pobreza y se dedicaban, especialmente, al servicio doméstico. Es hasta 1945 cuando se valoró su presencia en el proletariado marroquí, y la época de la Independencia de Marruecos marcó la diferencia en cuanto al trabajo femenino se trate, gracias a los cambios que conoció la sociedad marroquí durante los primeros y posteriores años de la Independencia: la escolarización de las niñas y el desarrollo de la educación, entre otros.

Dependiendo a qué contexto geográfico pertenecen, las mujeres de las zonas rurales realizan actividades, sea dentro o fuera del hogar familiar: trabajos poco reconocidos y no remunerados; mientras que las mujeres en el contexto urbano realizan empleos fuera de la economía familiar y del espacio doméstico, y los combinan con sus obligaciones familiares y del hogar.

En función del sector de trabajo -formal o informal-, se ve cuestionada la construcción del género. Las mujeres ocupadas en los sectores formales -trabajadoras cualificadas y cuyos sueldos son altos- hacen que se cuestionen los roles de género. De lo contrario, las actividades informales que realizan algunas mujeres en su hogar les permiten conseguir un sobresueldo para la unidad familiar, evitando, asimismo, cuestionar tales roles.

En la década del dos mil, la participación femenina en la actividad económica marroquí se inscribe en una tendencia ascendente. Sin embargo, la feminización de la función pública y el aumento del número de mujeres no implican la existencia de equidad e igualdad de 
oportunidades respecto al acceso a las responsabilidades y las funciones. Todavía existen obstáculos que impiden a las mujeres llegar a las esferas de toma de decisiones en igualdad con los hombres, a pesar de la existencia de las leyes que lo estipulan.

Los sectores donde están ocupadas las mujeres marroquíes son esencialmente la "agricultura, bosque y pesca”, “servicios” y la "industria”. Respecto al desempleo, las mujeres activas jóvenes y las universitarias se ven más sometidas al desempleo, lo que refleja la gran fragilidad en cuanto a su inserción laboral. De hecho, el perfil de las mujeres en situación de desempleo es caracterizado, esencialmente, por su edad joven y su cualificación. Por otra parte, las mujeres marroquíes trabajadoras presentan una tasa relativamente baja de sindicalización, sufren acoso sexual en el ámbito de trabajo y gran mayoría de ellas no se halla cubierta por el régimen del Seguro Médico Obligatorio.

La situación de desprestigio y los bajos sueldos del trabajo remunerado se han resuelto con las migraciones interiores y la emigración al extranjero de las mujeres. En el caso de las mujeres cualificadas, su emigración al extranjero deduce que la obtención de títulos y el reconocimiento de la igualdad de derechos civiles y políticos no bastan para garantizar su integración laboral.

Por último, todavía se asiste en Marruecos al fenómeno del trabajo infantil, incluido el de las niñas trabajadoras domésticas: “les petites bonnes”. Estas niñas suelen trabajar en condiciones de abuso y explotación. Ante esta situación a la que se ven sometidas, escapan del domicilio donde trabajan y se encuentran solas en la calle donde sufren todo tipo de violencia, lo que hace que sean futuras madres solteras.

\section{BIBLIOGRAFÍA}

Aixelá, Y. (2000) Mujeres en Marruecos. Un análisis desde el parentesco y el género.Barcelona: Bellaterra.

Ajaaouani, N. (2013). La realidad jurídica y social de les petites bonnes en Marruecos. disponible en http://www.revistacodice.es/publi_virtuales/v_congreso_mujeres/comunicaciones/la_realidad_juridica_y_social.pdf

Ajaaouani, N. (2012). Situación de la mujer en Marruecos tras las reformas del nuevo Código de Familia (Mudawwana, 2004). Una perspectiva histórico-social y jurídica. disponible en http:/www.revistacodice.es/publi_virtuales/iv_congreso_mujeres/comunicaciones/NAIMAI.pdf

Benlabbah, F (2008). Islam y derechos de la mujer en Marruecos. Cadernos Pagu, nº 30, pp. 95-106.

Chafai, L (1998). Las mujeres sujeto de marginación en Marruecos. Anales de Historia Contemporánea, $\mathrm{n}^{\circ} 13$, pp. 35-55. 
Comisión Española de Ayuda al Refugiado CEAR (2013). Marruecos. disponible en http:// cear.es/wp-content/uploads/2013/08/MARRUECOS.-2013.-Informe-general.

Díez Celaya, R. (1999). La mujer en el mundo. Madrid: Acento Editorial.

Diario de la Universidad Pablo de Olavide (22 de julio de 2010). Las mujeres están asumiendo responsabilidades políticas en Marruecos gracias a la discriminación positiva. disponible en http://web.upo.es/diario/2010/0722.htm Documento3

El Harras, M. (2004). La juventud marroquí ante el siglo XXI: cambios y desafíos. En López García, B. y Berriane, M. (dirs.), Atlas 2004 de la inmigración marroquĩ en España (pp.41-43). Madrid: TEIM.

Green, D. (2008). De la pobreza al poder: Cómo pueden cambiar el mundo ciudadanos activos y Estados eficaces. Barcelona: Ediciones Octaedro.

Haut-Commissariat au Plan (2015). Activité, emploi et chômage. disponible en http://www. hcp.ma/

Haut-Commissariat au Plan (2013). Femmes marocaines et marché du travail : caractéristiques et évolution. disponible en http://www.hcp.ma

Khachani, M. (2011). Genre et migration au Maroc. disponible en http://cadmus.eui.eu/ bitstream/handle/1814/15616/carim_asn_2011_19.pdf?sequence=1

Lahlou, M. (2001). Le travail des enfants au Maroc. Cadre macro-économique et social et données de base. disponible en http://www.erudit.org/livre/aidelf/2002/001340co.pdf

López Enamorado, M D (2003) Mujeres marroquíes en transición. Thémata Revista de Filosofía, $\mathrm{n}^{\circ} 31$, pp. 101-117.

Martín, I (2008). Mujer magrebí, joven, licenciada...y sin empleo. Afkar/Ideas, Revista trimestral para el diálogo entre el Magreb, España y Europa, nº 18, pp. 64-66.

Ramírez Fernández, Á. (1998). Migraciones, Género e Islam: mujeres marroquíes en España. Madrid: Agencia Española de Cooperación Internacional, Ediciones Mundo Árabe e Islam. Educación y Cultura.

Ramírez Fernández, Á. (2004). Mujeres en Marruecos: panorama social y jurídico. En López García, B. y Berriane, M. (dirs.), Atlas 2004 de la inmigración marroquí en España (pp. 37-40). Madrid: TEIM.

Solís Pérez, M (2010). La construcción simbólica de un mercado de trabajo feminizado en la ciudad de Tánger: una aproximación. Frontera Norte, vol. 22, nº 43, pp. 55-80.

Soriano Miras, R. M. (2004). El asentamiento de la mujer marroquí en el poniente almeriense. Madrid: Consejo Económico y Social. 
Análisis de la evolución de la situación... | Khadija Yahya

Soriano Miras, R M y Santos Bailón, C (2002). El perfil social de la mujer inmigrante marroquí en España y su incidencia en la relación intercultural. Papeles De Geografía, n ${ }^{\circ}$ 36, pp. 171-184.

Zirari, H. (2010). Los derechos de las mujeres en Marruecos: balance y perspectivas. disponible en http://www.fmyv.es/ci/es/mujer/61.pdf 